\title{
Evaluation of high volume extracapsular cataract extraction with posterior chamber lens implantation in Sierra Leone, west Africa
}

Kissy Eye Hospital, PO Box 115, Freetown, Sierra Leone N J Cook

Correspondence to: 26 North Road, Liverpool L19 OLR

Accepted for publication 1 April 1996

\begin{abstract}
Aims-Intraocular lens (IOP) implantation in sub-Saharan Africa is not the routine procedure of choice in the treatment of cataract. Previous series consist of small numbers of selected patients. The purpose of this study was to evaluate routine posterior chamber lens implantation in large numbers of consecutive patients. Methods-1059 cataract extractions with planned IOL (744 patients) were performed over a 1 year period from August 1993 to July 1994.

Results-94.6\% (1002) of eyes showed an improvement in visual acuity. A postoperative uncorrected acuity of $6 / 18$ or better was attained in $41.7 \%$ of eyes, while $27.1 \%$ attained an uncorrected acuity of $<6 / 18$ to 6/60. The commonest operative complication was posterior capsule rupture (11.4\%).

Conclusion-Extracapsular cataract extraction with posterior chamber lens implantation, performed in a well equipped clinic, can give satisfactory results in a developing country. Uncorrected visual acuity can be taken as an acceptable endpoint in the majority of cases.

(Br f Ophthalmol 1996;80:698-701)
\end{abstract}

There are an estimated 3.1 million people blind from cataract in sub-Saharan Africa. Extracapsular cataract extraction (ECCE) with posterior chamber lens implant (PC IOL) is the routine procedure of choice in industrialised countries, but is performed in relatively few centres in Africa. Consequently, there are few reports on the results of cataract surgery with intraocular lenses (IOLs) in Africa and only two reports from west Africa on 49 and 150 patients respectively. ${ }^{23}$ This study reports the experience on 1059 cataract extractions with planned IOL performed at Kissy Eye Hospital, Freetown, Sierra Leone.

\section{Methods}

IOL surgery has been performed in this hospital since 1983 with over 6000 posterior chamber lens implants operations undertaken under local anaesthesia as day cases. The centre has facilities for keratometry, ultrasound biometry, and microscopic surgery. There is a regular supply of intraocular lenses, viscoelastic substances, irrigating solutions, and microsurgical sutures. Lens implant power is calculated using the SRK 1 formula. In most cases the aim is a postoperative refractive error of -1 to -2 dioptres.

Postoperatively, patients are treated with topical steroid-antibiotic preparations for 2-4 weeks, and cycloplegia where indicated. At 4 weeks, visual acuity is measured and detailed examination of the anterior and posterior segments undertaken. If there is low acuity and/or the patient expresses a desire for better acuity, a refraction is performed. Sutures are removed if necessary, and spectacles prescribed where indicated. Many patients travel to Freetown from long distances. This causes problems with compliance with follow up.

A prospective study was undertaken between August 1993 and July 1994 to evaluate the results of extracapsular surgery with IOL implantation. Data from patients with known pre-existing ocular pathology likely to affect final visual outcome were excluded (for example, traumatic cataract, uveitic cataract, known glaucoma, pterygium, and corneal scars) During the study period, half the surgery was performed by the author, $27 \%$ by experienced visiting American ophthalmologists, and $23 \%$ by national ophthalmologists in training.

Visual acuity was measured using an Illiterate $\mathrm{E}$ chart. If it was worse than $6 / 60$, it was recorded as counting fingers (CF) 4 metres, CF 2 metres, hand movements, or perception of light. Visual status was categorised (Table 1) according to the WHO categories of visual impairment.

\section{Results}

PATIENT PROFILE

A total of 1059 extracapsular cataract extractions were performed on 744 patients. The average age at operation was 59.2 years (range 33 to 92$)$. A total of 926 operated eyes $(87 \%)$ were examined at 4 weeks; 133 eyes (13\%) were lost to follow up at varying times before the 4 week examination. The pre- and postoperative visual status of the patient is shown in

Table 1 WHO classification of visual impairment

\begin{tabular}{ll}
\hline Category of visual loss & $\begin{array}{l}\text { Corrected visual acuity in the } \\
\text { better eye }\end{array}$ \\
\hline 0 Normal vision & $6 / 18$ or better \\
1 Visual impairment & Less than $6 / 18$ to $6 / 60$ \\
2 Severe visual impairment & Less than $6 / 60$ to $3 / 60$ \\
3 Blind & Less than $3 / 60$ to $1 / 60$ \\
4 Blind & Less than $1 / 60$ to LP \\
5 Blind & NLP \\
\hline
\end{tabular}

Adapted from WHO. ${ }^{11}$ 
Table 2 Complications of ECCE/PC IOL surgery in 1059 eyes

\begin{tabular}{lcc}
\hline & No of eyes & $\%$ \\
\hline Operative: & & \\
Posterior capsule rupture, no IOL implantation & 112 & 10.6 \\
PC IOL placed, despite small PC tear & 8 & 0.76 \\
Insertion of AC IOL & 1 & 0.09 \\
Early postoperative (1-28 days): & 11 & 1.03 \\
Iris prolapse & 8 & 0.76 \\
Pupil capture & 5 & 0.47 \\
Pupillary inflammatory membrane & 4 & 0.38 \\
Persistent corneal oedema & 4 & 0.38 \\
Persistent soft lens matter & 3 & 0.28 \\
Early endophthalmitis, responsive to treatment & 2 & 0.19 \\
Fulminant endophthalmitis & 1 & 0.09 \\
Wound gape (resutured) & & \\
\hline
\end{tabular}

Figure 1. The pre and 4 week postoperative visual acuity in the operated eye is shown in Figure 2. Figure 2 indicates the acuity of all operated eyes, including the 133 eyes lost to follow up before the 4 week examination.

Altogether, $60.8 \%$ patients presented as blind or severely visually impaired. The $28.2 \%$ classified as normal visual status relates to unilateral or asymmetrical cataract. Postoperatively $59.9 \%$ had normal visual status: $91.7 \%$

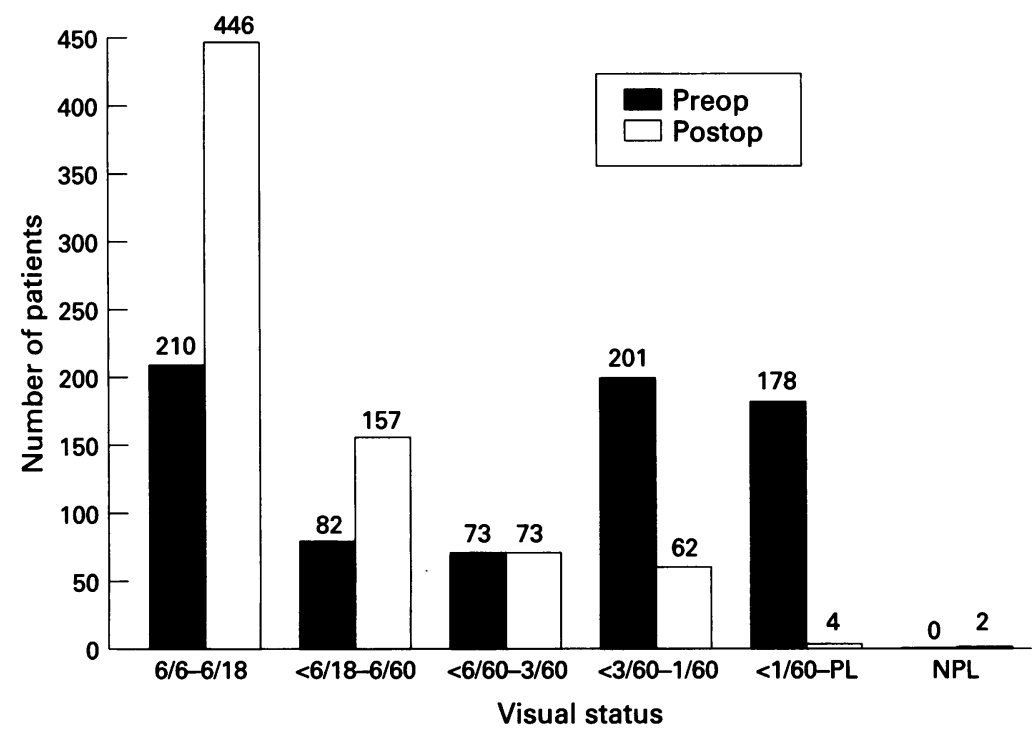

Figure 1 Visual status, pre and postoperative (at or before 4 weeks) in 744 patients undergoing planned ECCE/PC IOL surgery.

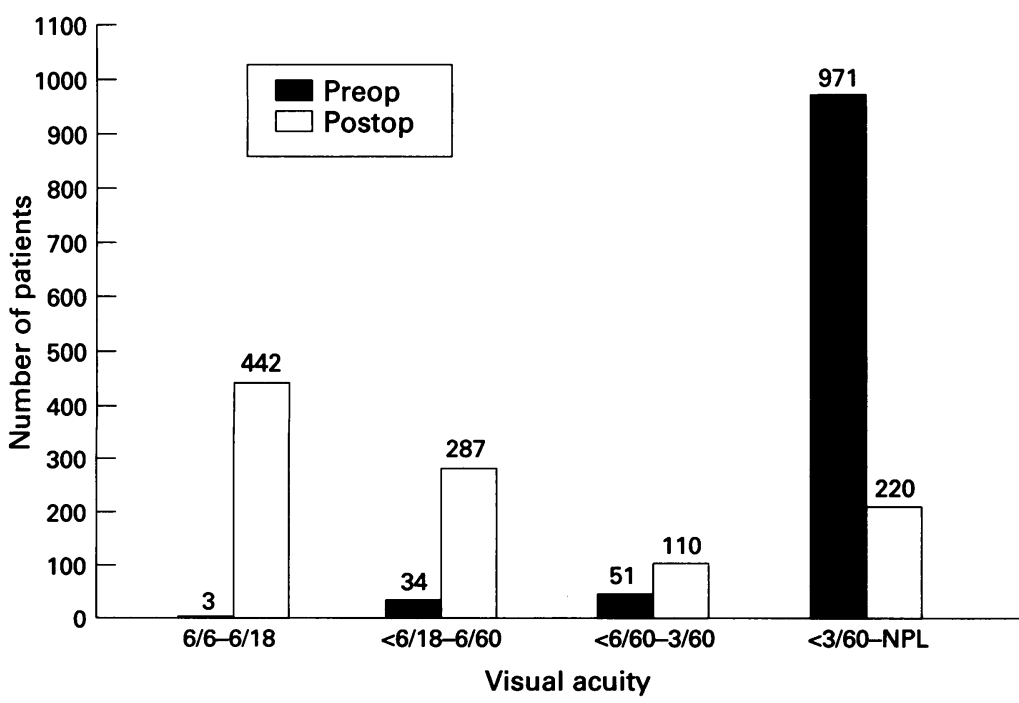

Figure 2 Visual acuity, pre and postoperative (at or before 4 weeks) in 1059 eyes undergoing planned ECCE/ PCIOL surgery.
Table 3 Causes of poor visual acuity $(<6 / 60)$ in 330 operated eyes out of 926 eyes seen at 4 weeks after ECCE surgery

\begin{tabular}{lrr}
\hline & $\begin{array}{l}\text { No of } \\
\text { eyes }\end{array}$ & $\%$ \\
\hline Surgical: & & \\
Uncorrected aphakia & 110 & 11.9 \\
Posterior capsule opacity & 18 & 1.9 \\
Known refractive error & 12 & 1.3 \\
Decentred IOL & 9 & 1.0 \\
Persisting corneal oedema & 6 & 0.6 \\
Soft lens matter remnant & 4 & 0.4 \\
Membrane around IOL & 3 & 0.3 \\
Fulminant endophthalmitis & 2 & 0.2 \\
Pre-existing pathology: & 18 & 1.9 \\
Optic atrophy & 16 & 0.5 \\
Posterior uveitis & 10 & 1.0 \\
Macular scar & 6 & 0.6 \\
Retinal detachment & 116 & 12.5 \\
Undetermined: & 330 & 34.1 \\
Total & & \\
\hline
\end{tabular}

of operated eyes had a preoperative acuity of $<3 / 60$. Only $3.5 \%$ had a preoperative acuity of $6 / 60$ or better. A postoperative uncorrected acuity of $6 / 18$ or better was attained in $41.7 \%$, while $27.1 \%$ cases attained acuity $<6 / 18-6 / 60$.

\section{COMPLICATIONS OF SURGERY}

These were subdivided into operative and postoperative (1-28 days), as shown in Table 2.

The commonest operative complication was posterior capsule rupture resulting in no IOL implantation (10.6\%). A further nine cases had IOL implantation despite a posterior capsule tear $(0.85 \%)$.

Iris prolapse was the commonest serious postoperative complication (1.03\%). There were five cases of endophthalmitis $(0.47 \%)$, of which three responded to treatment. In the other two cases the eye was lost.

CAUSES OF POOR VISUAL ACUITY $(<6 / 60)$ IN THE OPERATED EYE AT 4 WEEKS

A visual acuity at 4 weeks postoperatively of less than $6 / 60$ was considered a poor outcome. These cases, shown in Table 3 , were categorised into surgical, pre-existing pathology, or undetermined aetiology. Of the 926 eyes examined at 4 weeks, 330 eyes (35\%) were recorded as having a visual acuity of $<6 / 60$. Uncorrected aphakia was present in 110 eyes and previously undiagnosed pathology was found in 50 eyes. In 116 eyes the cause of poor acuity was not recorded.

MANAGEMENT OF REFRACTIVE ERRORS

Of the 45 pseudophakic patients $(6.0 \%)$ referred for formal refraction, 16 had glasses prescribed. One hundred of the 112 patients who did not receive an IOL had better acuity in the fellow eye and therefore aphakic spectacle correction was inappropriate; $57(50.8 \%)$ of these aphakic eyes had an IOL in the fellow eye and $43(38.4 \%)$ had a phakic fellow eye with good acuity. Of the 12 patients suitable for aphakic correction, nine had bilateral aphakia and three had poor acuity in the fellow phakic eye. Only two patients received corrective aphakic spectacles. 


\section{Discussion}

SURGICAL TECHNIQUE

The surgical technique in developing countries has to be adapted according to both limited resources and more advanced pathology. In our experience, approximately $50 \%$ of procedures can be carried out without the use of viscoelastic substance. The anterior capsulotomy is performed within a sealed anterior chamber and the implant can be inserted beneath air after partial closure of the section. However, a viscoelastic substance should always be available in case of complicated surgery or where air insertion fails on the first attempt.

In developing countries, large numbers of hypermature and Morgagnian cataracts are encountered resulting in a higher incidence of certain surgical problems. The extraction of Morgagnian cataracts, which have a small hard nucleus floating in a liquefied cortex, results in flooding of the anterior chamber with milky white fluid when the anterior capsule is first perforated. The poor visualisation and loss in capsule tension make the anterior capsulotomy more difficult to complete. In addition, as previously described, ${ }^{4}$ the lens capsules of hypermature cataracts are different from those of immature cataracts. Capsules of hypermature cataracts are often wrinkled, tough, or even calcified, and without elasticity. Gentle capsulotomy is difficult. This, combined with the recognised weaker zonules of hypermature cataracts, contributes to the greater risk of posterior capsule rupture and vitreous loss. Although some of these hazards can be reduced by appropriate surgical technique, they are a significant contributory factor in the $11.3 \%$ incidence of posterior capsule rupture. This contrasts with an incidence in the UK of $3.5 \%,{ }^{5}$ but is similar to two other African series of $10 \%$ in Ghana ${ }^{2}$ and $11 \%$ in Malawi. ${ }^{4}$

Posterior capsule rupture usually resulted in aphakia. However only 12 of 112 aphakic patients actually required aphakic spectacle correction because the majority had either good vision or were pseudophakic in the other eye. The fact that 10 of the 12 patients deemed suitable for aphakic correction were left uncorrected demonstrates that the rare bilateral aphakic patient can be easily overlooked in a busy clinic where pseudophakia is the rule. In several instances, the patient was lost to follow up at an early stage before the importance of correction could be emphasised.

VISUAL OUTCOME

Fifty one per cent of our patients came for surgery when they were blind in the better eye Similarly, Hennig et al in $\mathrm{Nepal}^{6}$ reported that approximately half of their cataract patients (1000 ICCEs) were blind by WHO definition: $91.7 \%$ of our patients had a preoperative visual acuity of less than $3 / 60$. This is similar to other studies in developing countries. Hennig et al found $91 \%$ patients with an acuity of $1 / 60$ or less. In Ghana (49 ECCE/PC IOL), all except one patient had an acuity of counting fingers or worse. ${ }^{2}$ In our series, the $3.5 \%$ of patients with a visual acuity of $6 / 60$ or better represent the few professional people with a low threshold for visual disability, usually presenting with reading difficulties.

Cataract extraction with intraocular lens implant improves the visual acuity in the majority of eyes undergoing the procedure. In this series, $94.6 \%$ (1002) of eyes showed an improvement in visual acuity; 53 eyes maintained the same acuity as a result of preexisting fundal pathology or operative aphakia and four eyes had a decrease in visual acuity, either due to endophthalmitis (two) or intraocular membrane formation (two). In the series in Ghana, ${ }^{2}$ where direct comparison with uncorrected acuity is possible, $84 \%$ showed an improvement in acuity, $10 \%$ remained the same, and in $6 \%$ the acuity worsened.

In this study, 4 weeks was taken as the end point for assessing visual acuity. However, this does not represent the true final result for a significant number of patients. Four weeks was used in order to obtain a reasonable percentage for follow up. Many patients at 4 weeks are still on cycloplegics and ointments which reduce acuity. Some are awaiting removal of tight sutures. The visual acuities in some patients may improve with time.

Follow up is always a problem in Africa; $87 \%$ of our patients kept their appointments at 4 weeks. This is remarkable in view of the difficulties in transport and communication affecting most of the country.

Some $41.7 \%$ of patients had an uncorrected visual acuity of $6 / 18$ or better at 4 weeks, with $27.1 \%$ having an acuity between $6 / 60$ and $6 / 18$. Comparison of average uncorrected visual acuity with other studies is limited, since most other authors, even in developing countries, consider best corrected visual acuity as the endpoint of a cataract extraction. However, despite these limitations, our results can be compared with those series which provide figures for uncorrected visual acuity. In $\mathrm{Nepal}$, Ruit et $a l^{7}$ reported $47.9 \%$ of patients with uncorrected acuity of $6 / 15$, with $40 \%$ having an acuity of between $6 / 60$ and 6/18. In Ghana ${ }^{2}$ average unaided acuity was reported at between $6 / 24$ and 6/30. Biometry was not used in the series in Nepal and Ghana. This limits direct comparison with our results where biometry was performed routinely and a small degree of myopia was the planned outcome. In the UK, Aylward et $a l^{5}$ report an average unaided acuity of between $6 / 18$ and $6 / 24$.

The best corrected visual acuity was deliberately not taken as the end point in this study, in contrast with others, because in most cases patients would not buy spectacle correction if they were satisfied with their unaided acuity.

The practice at Kissy for pseudophakics is to allow the patients to decide their own need for glasses. If they are satisfied with their near and distance vision, and their distance acuity is better than $6 / 60$, then they are discharged. If the level of acuity is not acceptable to the patient or is $6 / 60$ or worse, anterior and posterior segments are examined and refraction performed if indicated. As a result of these criteria, only $45(4.2 \%)$ patients required refraction and only $16(1.5 \%)$ received spectacle correction either because the necessary 
high cylinder correction was not available or because the patient decided that he could not afford the spectacles. These figures contrast with the series from Ghana where refraction was mandatory and free spectacles dispensed. The policy at Kissy is to aim for overcorrection to produce a refractive error of -1 to -2 dioptres. In this way, most patients attain functional uncorrected near acuities (Jaeger 5-6), and although many patients comment that their distance acuity is not as sharp as the near, they are tolerant and accepting of a distance acuity that is less than perfect. Under ideal circumstances, a patient questionnaire might have been used to evaluate this conclusion and confirm whether planned myopia was desirable. We would accept that the patients' perception, in combination with actual recorded acuity, might be considered a crude form of assessment of need for refraction. However, our optometrist was unable to refract all pseudophakics routinely and the majority of patients could not afford the costs of spectacles, even when available and subsidised. The refractive workload after implanting intraocular lenses can, therefore, be small compared with the workload of providing all patients with aphakic correction.

It has previously been stated that less than optimal postoperative visual acuity in developing countries is due to coexisting disease, intraoperative complications, and imperfect refractive correction. ${ }^{89}$ Our study supports this statement. An acuity of $<6 / 60$ was used as an indicator of poor outcome. One hundred and twelve patients were aphakic because an IOL could not be used, but only 12 of these had poor vision in the fellow eye. In 116 patients, the reason for low acuity was not recorded. This was because they escaped rigorous examination by the author himself. These incomplete data, although unfortunate, reflect that the author had responsibility for mobile clinics beyond Freetown and relied on his deputy for some data collection. It is unlikely that any of these patients had important complications such as endophthalmitis because they were all examined by other ophthalmologists. In some of these cases the posterior segment was not apparently examined and pre-existing disease was not excluded. In other cases, the notes recorded that the patient was pleased with the result and a search was not undertaken to assess the posterior segment or refractive status. This would support our overall clinical impression that the actual acuity was often better than $6 / 60$, and that some patients are unable to comprehend the Illiterate $E$ charts. The limitations of accurately measuring visual acuity should not be underestimated in a society where multiple tribal languages make communication between fellow nationals difficult. Additionally, some of these patients were still on cycloplegics or ointments which could reduce the acuity.

We were not able to select patients entirely free from pre-existing disease because the posterior segment could not be visualised. This contributed to poor postoperative visual acuity in 50 patients. Pre-existing posterior uveitis reflects the high prevalence of ocular toxoplasmosis in the population. Optic atrophy was usually secondary to onchocerciasis, which is endemic in parts of the country. Five cases of endophthalmitis occurred and in two cases there was no response to treatment and the eye was lost. This incidence of endophthalmitis $(0.47 \%)$ is similar to that found in developed countries. Two recent series in the UK report an incidence of $0.3 \%{ }^{10}$ and $058 \% .^{5}$

Eighteen eyes were considered to have posterior capsule opacity at 4 weeks. No conclusions can be drawn from this. Late posterior capsule opacification could not be determined because long term follow up is impractical. Most patients travel long distances for surgery and are unlikely to return unless acuity falls below navigating vision. Even in these circumstances, the financial means to return is often not available. The rate of late posterior capsule opacification remains an important unresolved question regarding the suitability of extracapsular surgery in the developing world.

This study shows that extracapsular cataract extraction with posterior chamber implant, performed in a well equipped clinic, can give satisfactory results in a developing country. Our series is unique in that it involves large numbers of consecutive patients who underwent full biometry where all lens implant powers were available.

1 WHO. Available data on blindness (Update 1987). Geneva: WHO/PBL/87.14, 1987.

2 Egbert PR, Buchanan M. Results of extracapsular cataract surgery and intraocular lens implantation in Ghana. Arch Ophthalmol 1991;109:1764-8.

3 Borzeix A, Rivaud C, Lam A, Ancel JM, Ruffin M, Faye M, Sechs CM. L' implantation de chambre posterieure en Afrigue noir. A propos de 150 cas suivis pendant 18 mois. f Fr Ophtalmol 1993;16:663-7.

4 Lewallen S, Le Measurier RT. Extracapsular cataract extraction in developing countries. [Letter] Arch Ophthalmol 1993;111:18-9.

5 Aylward G, Larkin D, Cooling R. Audit of cost and clinical outcome of cataract surgery. Health Trends 1993;25:126-9.

6 Hennig A, Shrestha SP, Foster A. Results and evaluation of high volume intracapsular cataract surgery in Nepal. Acta Ophthalmol 1992;70:402-6.

7 Ruit S, Robin AL, Pokhrel RP, Sharma A, De Faller J. Extracapsular cataract extraction in Nepal. Arch Ophthalmol 1991;109:1761-3.

8 Hemo I. Intraocular lens implantation in an underdeveloped country. $f$ Cataract Refract Surg 1987;13:414-6.

9 Pe'er J, Wood M. Intraocular lens implantation in developing countries. $\mathcal{f}$ Cataract Refract Surg 1990;16:621-3.

10 Desai P. The National Cataract Surgery Survey: II. Clinical outcomes Eye 1993;7:489-94.

11 WHO. Guidelines for programmes for the prevention of blindness. Geneva: World Health Organisation, 1979. 